\title{
Global in time solutions for the Poiseuille flow of Oldroyd type in 3D domains
}

\author{
B. Climent Ezquerra and F. Guillén GonzÁlez *
}

\begin{abstract}
A Poiseuille flow in a 3D cylindrical domain is considered for a non-newtonian fluid of Oldroyd type.

We prove existence (and uniqueness) of a global (in time) weak solution. Moreover, this weak solution is an strong solution when data are more regular. These results has already been obtained in the case of two concentrical cylinders ([3]).

Now, we consider an extension to an unique cylinder. Then, a mixed parabolic-hyperbolic PDE's system appears but the parabolic equation is of degenerate type. The key of the proofs is to estimate in appropriate Sobolev weighted spaces (and to obtain strong convergence in weak norms by means of a Cauchy argument).
\end{abstract}

Abbreviated Title: Global solutions for the Poiseuille flow of Oldroyd type.

AMS subject Classification: 35K65, 35Q30, 76A10, 76D05.

Keys Words: Poiseuille flow, Oldroyd law, degenerate parabolic equations, Weigthed Sobolev spaces, Non-newtonian fluid.

\footnotetext{
*Department of Differential Equations and Numerical Analysis, Universidad de Sevilla, Aptdo. 1160, 41080 Sevilla, Spain, E-mails: blanca@numer.us.es, guillen@numer.us.es.
} 


\section{Introduction}

In this paper we consider a Poiseuille flow for an incompressible viscoelastic tridimensional fluid satisfying the Oldroyd constitutive law $([1],[6])$. Then, we consider the fluid confined in a cylinder

$$
\Omega(0, R)=\{(r, \varphi, z): 0<r<R, 0 \leq \varphi<2 \pi, z \in \mathbb{R}\},
$$

in which an axisymmetric motion is assumed.

If one use cylindrical coordinates, after some simplifications, one can reduce the problem in $\Omega(0, R) \times(0, T)$ to the follow differential system in $(0, R) \times(0, T)$ (see [7],[3] for details of derivation of the model):

$$
\begin{aligned}
\operatorname{Re} \partial_{t} v-(1-\alpha) r^{-1}\left(r v_{r}\right)_{r} & =r^{-1}\left(r \sigma_{2}\right)_{r}+f, \\
\partial_{t} \sigma_{1}+\mathrm{We}^{-1} \sigma_{1} & =\left(1-a^{2}\right) \sigma_{2} v_{r}, \\
\partial_{t} \sigma_{2}+\mathrm{We}^{-1} \sigma_{2} & =\alpha \mathrm{We}^{-1} v_{r}-\sigma_{1} v_{r} .
\end{aligned}
$$

Here, $\partial_{t}$ denotes the $t$-partial derivate whereas the $r$-partial derivate will we denoted by the subindex $r$. Re is the reynolds number (the ratio between inertial and viscous forces acting on the fluid) and We is the Weissenberg number (a messure of the elasticity of the fluid). The constants $\alpha$ and $a$ verify $0<\alpha<1,|a|<1$. We will complete (1)-(3) with appropriate boundary and initial conditions. The unknows are $v, \sigma_{1}$ and $\sigma_{2}$ which depend on the independent variables $(r, t) \in(0, R) \times(0, T)$. The velocity of the fluid in the $z$-direction is modelled by $v=v(r, t)$, whereas $\sigma_{1}, \sigma_{2}$ represent linear combinations of the some extra-stress tensor components.

Theoretical results for the general $3 D$ Oldroyd model (and other viscoelastic models) can be viewed in [8], [4], [3]. Global existence and uniqueness for the 2D Poiseuille flow has been proved in [5]. With respect to 3D case, in [3] is deduced the global existence in time and uniqueness of the problem (1)-(3) in $\left(R_{1}, R_{2}\right) \times(0, T)$, whenever $0<R_{1}<r<R_{2}$ (i.e., the fluid is confined between two concentrical cylinders) jointly homogeneous boundary conditions on $r=R_{1}$ and $r=R_{2}$. Now, the main difficulty is that we have not an upper bound for $r^{-1}$. In this sense, (1) is a parabolic equation of degenarate type. Moreover, it is not clear which boundary condition on $r=0$ must be imposed (it will appear a Neumann condition).

The main task in this paper is to solve $(1)-(3)$ in $(0, T) \times(0, R)$ together with the following boundary and initial conditions:

$$
\begin{gathered}
v(R, t)=0, \quad v_{r}(0, t)=0 \quad \text { for } t \in(0, T), \\
\left.v\right|_{t=0}=v_{0},\left.\quad \sigma_{i}\right|_{t=0}=\sigma_{i, 0} \quad(i=1,2) \quad \text { in }(0, R) .
\end{gathered}
$$

This paper is organized as follows. In Section 2, we enonce the two main results of this paper, existence and uniqueness of weak solution of (1)-(3) (Theorem 1) and regularity of this weak solution when data are more regular (Theorem 2). In Section 3, we prove technical results about some weighted space of functions, where we will find the solution. The proof of Theorem 2 is made 
in Section 4, using a semi-Galerkin approach together with strong estimates that let us pass to the limit by compactness. Finally, in Section 5 we prove Theorem 1, obtaining strong convergence in weak norms of a sequence of approximate regular solutions (furnished by Theorem 2) by means of a Cauchy argument.

\section{The main results}

To give a variational formulation of problem (1)-(5), we define the following Hilbert spaces

$$
\begin{aligned}
H_{R}^{1} & =\left\{v: v \in H^{1}(0, R), v(R)=0\right\}, \\
H & =\left\{v: r^{-1 / 2} v \in L^{2}(0, R)\right\}, \\
V & =\left\{v: r^{1 / 2} v \in L^{2}(0, R), r^{1 / 2} v_{r} \in L^{2}(0, R), v(R)=0\right\}, \\
W & =\left\{\sigma: r^{-1 / 2} \sigma \in L^{2}(0, R), r^{-1 / 2}(r \sigma)_{r} \in L^{2}(0, R)\right\} .
\end{aligned}
$$

We will denote $H_{R}^{-1}, H^{\prime}$, and $V^{\prime}$, as the dual space of $H_{R}^{1}, H$ and $V$, respectively. The norm and the scalar product in $L^{2}(0, R)$ will be denoted by $\|\cdot\|$ and $(\cdot, \cdot)$, respectively. The notation for the space of functions will be abreged. For instance, $L^{2}=L^{2}(0, R)$ or $H^{1}=H^{1}(0, R)$. Whenever $X(0, R)$ is a Banach space formed by functions depend on $(0, R), L^{p}(X)$ stands for $L^{p}(0, T ; X(0, R))$ and $C(X)$ stands for $C([0, T] ; X(0, R))$.

Theorem 1 (Existence and uniqueness of weak solution) Assume that $T>0,|a|<1$, $0<\alpha<1$. If $v_{0} \in H, \sigma_{i, 0} \in L^{\infty}(i=1,2)$ and $r f \in L^{2}\left(V^{\prime}\right)$, then (1)-(5) possesses one unique weak solution $\left\{v, \sigma_{1}, \sigma_{2}\right\}$ in $(0, R) \times(0, T)$, that is

$$
\begin{gathered}
v \in L^{2}(V), \quad r^{1 / 2} v \in L^{\infty}\left(L^{2}\right), \quad \partial_{t}\left(r^{1 / 2} v\right) \in L^{2}\left(H_{R}^{-1}\right), \\
\sigma_{i} \in L^{\infty}\left(L^{\infty}\right), \quad \partial_{t}\left(r^{1 / 2} \sigma_{i}\right) \in L^{2}\left(L^{2}\right) \quad i=1,2,
\end{gathered}
$$

equation (1) and the Neumann boundary condition on $r=0$ of (4) are satisfied in the following weak sense:

$$
\begin{aligned}
& \operatorname{Re}\left(\partial_{t}\left(r^{1 / 2} v\right), r^{1 / 2} w\right)+(1-\alpha)\left(r^{1 / 2} v_{r}, r^{1 / 2} w_{r}\right) \\
& \quad=-\left(r^{1 / 2} \sigma_{2}, r^{1 / 2} w_{r}\right)+\langle r f, w\rangle_{V^{\prime}, V} \quad \forall w \in V, \text { a.e. in }(0, T),
\end{aligned}
$$

equations (2)-(3) are satisfied a.e. in $(0, R) \times(0, T)$ and the initial conditions (5) are satisfied as

$$
\left.\left(r^{1 / 2} v\right)\right|_{t=0}=r^{1 / 2} v_{0},\left.\quad\left(r^{1 / 2} \sigma_{i}\right)\right|_{t=0}=r^{1 / 2} \sigma_{i, 0} \quad(i=1,2) .
$$

Notice that, the above initial conditions have a sens, because the (weak) regularity of $r^{1 / 2} v$ and $r^{1 / 2} \sigma_{i}$ implies in particular:

$$
r^{1 / 2} v \in C\left([0, T], H_{R}^{-1}\right), \quad r^{1 / 2} \sigma_{i} \in C\left([0, T], L^{2}\right) \quad(i=1,2) .
$$


Theorem 2 (Regularity) Under hyphotesis of Theorem 1, if moreover $v_{0} \in V, \sigma_{i, 0} \in W(i=$ $1,2)$ and $r^{1 / 2} f \in L^{2}\left(L^{2}\right)$, then the weak solution $\left\{v, \sigma_{1}, \sigma_{2}\right\}$ of $(1)-(5)$ is also a strong solution, that is, one has the additional regularity properties:

$$
\begin{gathered}
r^{1 / 2} v_{r} \in L^{\infty}\left(L^{2}\right) \cap L^{2}\left(H^{1}\right), \quad v_{r} \in L^{2}(W), \quad \partial_{t} v \in L^{2}\left(H^{\prime}\right) \\
\sigma_{i} \in L^{\infty}(W), \quad \partial_{t}\left(r^{-1 / 2} \sigma_{i}\right) \in L^{2}\left(L^{2}\right), \quad(i=1,2),
\end{gathered}
$$

equation (1) is verified a.e. in $(0, R) \times(0, T)$, the Neumann boundary condition on $r=0$ has the following sens of trace $\left.\left(r^{1 / 2} v_{r}\right)\right|_{r=0}=0$, and one verifies the initial conditions $\left.v\right|_{t=0}=v_{0}$ and $\left.\sigma_{i}\right|_{t=0}=\sigma_{i, 0}$ (now, $v \in C\left(H^{\prime}\right)$ and $\left.\sigma_{i} \in C\left(L^{2}\right)\right)$.

Remark: $H$ and $V$ are spaces of velocities $(v)$ and $W$ is the space of tensors $\left(v_{r}, \sigma_{1}\right.$ and $\left.\sigma_{2}\right)$.

\section{Some technical results}

Lemma $3 V$ is a Hilbert space endowed with the scalar product $\left(r^{1 / 2} v_{r}, r^{1 / 2} w_{r}\right)$, which is equivalent to the scalar product $\left(r^{1 / 2} v, r^{1 / 2} w\right)+\left(r^{1 / 2} v_{r}, r^{1 / 2} w_{r}\right)$. In fact, the embedding $V \hookrightarrow L^{2}(0, R)$ is continuous, verifying

$$
\|v\| \leq 2\left\|r v_{r}\right\| \quad \forall v \in V
$$

(in particular, $\left\|r^{1 / 2} v\right\| \leq R^{1 / 2}\|v\| \leq 2 R^{1 / 2}\left\|r v_{r}\right\| \leq 2 R\left\|r^{1 / 2} v_{r}\right\|$ ). Moreover, if $v \in V$ then $r v \in H^{1}$ (and therefore, $v(R)=0$ has a sens of trace).

PROOF:

We can write

$$
v(r)^{2}=-2 \int_{r}^{R} v(s) v_{r}(s) d s .
$$

Integrating in $r \in(0, R)$ and using Fubini's Theorem and Hölder's inequality, we obtain

$$
\|v\|^{2}=-2 \int_{0}^{R} v(s) s v_{r}(s) d s \leq 2\|v\| \cdot\left\|r v_{r}\right\| .
$$

Therefore, (6) holds. The rest of conclusions of Lemma are easy to obtain.

Lemma $4 W$ is a Hilbert space endowed with the scalar product

$$
\left(r^{-1 / 2}(r \sigma)_{r}, r^{-1 / 2}(r \chi)_{r}\right)
$$

which is equivalent to the scalar product

$$
\left(r^{-1 / 2} \sigma, r^{-1 / 2} \chi\right)+\left(r^{-1 / 2}(r \sigma)_{r}, r^{-1 / 2}(r \chi)_{r}\right)
$$

In fact, one has

$$
\left\|r^{-1 / 2} \sigma\right\| \leq\left\|r^{-1 / 2}(r \sigma)_{r}\right\| \quad \forall \sigma \in W .
$$


Moreover, if $\sigma \in W$, then $r^{1 / 2} \sigma_{r} \in L^{2}$ and $r^{1 / 2} \sigma \in H^{1}$, verifying

$$
\begin{gathered}
\left\|r^{1 / 2} \sigma_{r}\right\| \leq 2\left\|r^{-1 / 2}(r \sigma)_{r}\right\|, \\
\left\|\left(r^{1 / 2} \sigma\right)_{r}\right\| \leq 3\left\|r^{-1 / 2}(r \sigma)_{r}\right\| .
\end{gathered}
$$

Proof:

We write

$$
\left(r^{-1 / 2} \sigma(r)\right)^{2}=\left(R^{-1 / 2} \sigma(R)\right)^{2}-2 \int_{r}^{R} s^{-1 / 2} \sigma(s)\left(s^{-1 / 2} \sigma(s)\right)_{s} d s .
$$

Integrating in $(0, R)$, applying Fubini's Theorem and using that

$$
r\left(r^{-1 / 2} \sigma\right)_{r}=r^{-1 / 2}(r \sigma)_{r}-\frac{3}{2} r^{-1 / 2} \sigma
$$

we obtain

$$
2\left\|r^{-1 / 2} \sigma\right\|^{2}=-(\sigma(R))^{2}+2 \int_{0}^{R} r^{-1 / 2} \sigma(r) r^{-1 / 2}(r \sigma(r))_{r} d r .
$$

Therefore (7) holds. Taking into account that

$$
r^{1 / 2} \sigma_{r}=r^{-1 / 2}(r \sigma)_{r}-r^{-1 / 2} \sigma,
$$

it follows (8) thanks to (7). ¿From (7) and (8), we have (9).

Finally, the following regularity result we will also be used.

Lemma 5 If $(r \sigma)_{r} \in L^{2}(0, R)$ then $\sigma \in L^{2}(0, R)$ and

$$
\|\sigma\| \leq 2\left\|(r \sigma)_{r}\right\|
$$

PROOF:

We can write

$$
\sigma(r)^{2}=\sigma(R)^{2}-2 \int_{r}^{R} \sigma(s) \sigma_{r}(s) d s
$$

Integrating in $r \in(0, R)$ and applying Fubini's Theorem, we obtain

$$
\|\sigma\|^{2}=R \sigma(R)^{2}-2 \int_{0}^{R} r \sigma(r) \sigma_{r}(r) d r .
$$

Using that $r \sigma \sigma_{r}=(r \sigma)_{r} \sigma-\sigma^{2}$, then

$$
\|\sigma\|^{2}=R \sigma(R)^{2}+2\|\sigma\|^{2}-2 \int_{0}^{R}(r \sigma)_{r} \sigma d r .
$$

This implies

$$
\|\sigma\|^{2} \leq 2 \int_{0}^{R}(r \sigma)_{r} \sigma d r
$$

and thanks to Hölder's inequality, we obtain $\|\sigma\|^{2} \leq 2\left\|(r \sigma)_{r}\right\| \cdot\|\sigma\|$ and (10) holds.

First, we will prove Theorem 2. Afterwards, this result will be used in the proof of Theorem 1. 


\section{The proof of Theorem 2}

In the sequel, $C, C_{1}, C_{2}$ denote different constants which depend only on the data. We split the proof in three steps: the choice of a special basis of $V$, construction of a family of aproximate solutions by a Galerkin method and finally, the pass to the limit by compactness.

\section{FIRST STEP: The choice of a special basis of $V$.}

Let us consider the eigenvalue problem:

$$
\left\{\begin{array}{l}
\text { Obtain } \lambda \in \mathbb{R} \text { and } v \in V(v \neq 0), \text { such that } \\
r^{-1}\left(r v_{r}\right)_{r}=\lambda v \quad \text { in }(0, R), \\
v_{r}(0)=0, \quad v(R)=0 .
\end{array}\right.
$$

A variational formulation of (11) is (see [2]):

$$
\begin{cases}\text { Obtain } \lambda \in \mathbb{R} \text { and } v \in V(v \neq 0), & \text { such that } \\ \left(r^{1 / 2} v_{r}, r^{1 / 2} w_{r}\right)=\lambda\left(r^{1 / 2} v, r^{1 / 2} w\right) & \forall w \in V .\end{cases}
$$

¿From Lemma 3, the bilinear form defined by

$$
\left(r^{1 / 2} u_{r}, r^{1 / 2} v_{r}\right) \quad \forall u, v \in V
$$

defines in $V$ an scalar product, that it will be denoted by $(\cdot, \cdot)_{V}$ (and by $\|\cdot\|_{V}$ his corresponding norm).

For a fixed $u \in V$, the map $v \in V \mapsto\left(r^{1 / 2} u, r^{1 / 2} v\right) \in R$ is a continuous linear functional on $V$. So that, by the Riesz-Fréchet representation Theorem, there is a unique function in $V$, that it will be denoted by $T u$, such that

$$
(T u, v)_{V}=\left(r^{1 / 2} u, r^{1 / 2} v\right) \quad \forall v \in V .
$$

Clearly $T: V \mapsto V$ is linear, symmetric and bounded (in fact, $\|T u\|_{V} \leq\|u\|_{V}$ ). Moreover, $T$ is compact. Indeed, let $u_{n}$ be a bounded sequence in $V$, then in particular by Lemma $3, r u_{n}$ is bounded in $H^{1}$. Therefore, we may extract a subsequence, such that $r u_{n} \rightarrow r u$ strongly in $L^{2}$. Then,

$$
\left\|T\left(u_{n}-u\right)\right\|_{V}^{2}=\left(r\left(u_{n}-u\right), T\left(u_{n}-u\right)\right) \leq\left\|r\left(u_{n}-u\right)\right\| \cdot\left\|T\left(u_{n}-u\right)\right\|,
$$

which implies that $T u_{n} \rightarrow T u$ strongly in $V$.

Variational problem (12) may be rewritten as

$$
(u, v)_{V}=\lambda(T u, v)_{V} \quad \forall v \in V .
$$

Therefore, the problem is equivalent to

Obtain $\mu \in \mathbb{R}(\mu=1 / \lambda)$ and $u \in V$ such that $T u=\mu u$. 
Now, we can apply Hilbert-Schmidt's Theorem to obtain a sequence $\left(\mu_{n}\right)$ of eigenvalues of $T$ (with $\mu_{n}>0$ and $\left.\mu_{n} \nearrow \infty\right)$ and an orthonormal basis $\left(u^{n}\right)$ formed by eigenfunctions of $V$. Therefore, we have that the sequence $\lambda_{n}=1 / \mu_{n}\left(\lambda_{n}>0\right.$ and $\left.\lambda_{n} \searrow 0\right)$, is formed by "eigenvalues" of problem (12) associated to the same orthonormal basis $\left(u^{n}\right)$ of $V$

\section{SECOND STEP: Construction of a family of approximate solutions.}

We use a Galerkin approximation for $v$, remaining problems for $\sigma_{1}, \sigma_{2}$ at infinity dimension (this can be called a semi-Galerkin method) .

The finite dimensional space spanned by $\left\{u^{1}, u^{2}, \ldots, u^{m}\right\}$ will be denoted by $V^{m}$. For each $m \geq 1$, we define $\left(v^{m}, \sigma_{1}^{m}, \sigma_{2}^{m}\right)$ as an $m$-th approximate solution if

$$
\begin{gathered}
v^{m} \in C^{1}\left([0, T], V^{m}\right), \quad\left(\text { i.e. } v^{m}(t)=\sum_{i=1}^{m} \beta_{i}(t) u^{i} \text { with } \beta_{i} \in C^{1}[0, T]\right), \\
\sigma_{i}^{m} \in C\left([0, T], L^{\infty}(0, R)\right) \cap C^{1}\left([0, T], L^{2}(0, R)\right) \quad i=1,2,
\end{gathered}
$$

verifying the (approximate) problem:

$$
\begin{aligned}
& \operatorname{Re}\left(\partial_{t}\left(r^{1 / 2} v^{m}\right), r^{1 / 2} w\right)+(1-\alpha)\left(r^{1 / 2} v_{r}^{m}, r^{1 / 2} w_{r}\right) \\
& \quad=-\left(r^{1 / 2} \sigma_{2}^{m}, r^{1 / 2} w_{r}\right)+\left(r^{1 / 2} f_{m}, r^{1 / 2} w\right) \quad \forall w \in V^{m}, \\
& \partial_{t} \sigma_{1}^{m}+\mathrm{We}^{-1} \sigma_{1}^{m}=\left(1-a^{2}\right) \sigma_{2}^{m} v_{r}^{m}, \\
& \partial_{t} \sigma_{2}^{m}+\mathrm{We}^{-1} \sigma_{2}^{m}=\alpha \mathrm{We}^{-1} v_{r}^{m}-\sigma_{1}^{m} v_{r}^{m}, \\
& \left.v^{m}\right|_{t=0}=v_{0}^{m},\left.\quad \sigma_{i}^{m}\right|_{t=0}=\sigma_{i, 0}, \quad i=1,2,
\end{aligned}
$$

where $f_{m} \in C\left([0, T], L^{2}\right)$ such that $r^{1 / 2} f_{m} \rightarrow r^{1 / 2} f$ in $L^{2}\left(L^{2}\right)$. In (16), $v_{0}^{m}=P_{m}\left(v_{0}\right)$, taking $P_{m}: L^{2}(0, R) \mapsto V^{m}$ as the orthogonal projector onto $V^{m}$. Observe that $v_{0}^{m} \rightarrow v_{0}$ in $L^{2}$ (since $v_{0} \in V$, in particular $v_{0} \in L^{2}$, thanks to Lemma 3 ).

Now, for each $m \geq 1$, we are going to prove the existence of a solution $\left(v^{m}, \sigma_{1}^{m}, \sigma_{2}^{m}\right)$ of (13)-(16), by means of a fixed point argument (using linearisation of (13)-(16)).

Given $\bar{v}^{m} \in C\left([0, T], V^{m}\right)$, we stand the following linear differential problem in $(0, T) \times(0, R)$ : Obtain $\left(\sigma_{1}^{m}, \sigma_{2}^{m}\right) \in C^{1}\left([0, T] ; L^{2}\right)$ such that

$$
\begin{aligned}
& \partial_{t} \sigma_{1}^{m}+\mathrm{We}^{-1} \sigma_{1}^{m}=\left(1-a^{2}\right) \sigma_{2}^{m} \bar{v}_{r}^{m}, \\
& \partial_{t} \sigma_{2}^{m}+\mathrm{We}^{-1} \sigma_{2}^{m}=\alpha \mathrm{We}^{-1} v_{r}^{m}-\sigma_{1}^{m} \bar{v}_{r}^{m} \\
& \left.\sigma_{i}^{m}\right|_{t=0}=\sigma_{i, 0}, \quad i=1,2 .
\end{aligned}
$$


This problem can be written as the following Cauchy problem, associated to the linear differencial system respect to $t$ :

$$
\left\{\begin{array}{l}
\partial_{t} \sigma^{m}+A \sigma^{m}=b, \quad t \in(0, T), \quad \text { a.e. } r \in(0, R), \\
\left.\sigma^{m}\right|_{t=0}=\sigma_{0}, \quad i=1,2
\end{array}\right.
$$

where

$$
\sigma^{m}=\left(\begin{array}{c}
\sigma_{1}^{m} \\
\sigma_{2}^{m}
\end{array}\right), \quad A=\left(\begin{array}{cc}
\mathrm{We}^{-1} & -\left(1-a^{2}\right) \bar{v}_{r}^{m} \\
\bar{v}_{r}^{m} & \mathrm{We}^{-1}
\end{array}\right), \quad b=\left(\begin{array}{c}
0 \\
\alpha \mathrm{We}^{-1} \bar{v}_{r}^{m}
\end{array}\right) .
$$

As $\bar{v}^{m}$ is generated by $\left\{u^{1}, u^{2}, \ldots, u^{m}\right\}$, in particular we have that $\left(r \bar{v}_{r}^{m}\right)_{r} \in C\left([0, T] ; L^{2}\right)$, hence Lemma 5 says us that $\bar{v}_{r}^{m} \in C\left(L^{2}\right)$. So that, $A, b \in C\left([0, T] ; L^{2}\right)$ and we can deduce that (17)-(18) possesses one unique maximal solution in $C^{1}\left([0, T] ; L^{2}\right)$.

Now, given $\bar{v}^{m} \in C\left(V^{m}\right)$ and $\sigma_{i}^{m} \in C^{1}\left(L^{2}\right), i=1,2$, the solution of (17)-(19), we stand the linear variational problem: Obtain $v^{m} \in C^{1}\left([0, T], V^{m}\right)$ such that

$$
\left\{\begin{array}{l}
\operatorname{Re}\left(\partial_{t}\left(r^{1 / 2} v^{m}\right), r^{1 / 2} w\right)+(1-\alpha)\left(r^{1 / 2} v_{r}^{m}, r^{1 / 2} w_{r}\right)=-\left(r^{1 / 2} \sigma_{2}^{m}, r^{1 / 2} w_{r}\right) \\
\quad+\left(r^{1 / 2} f_{m}, r^{1 / 2} w\right) \quad \forall w \in V^{m}, \\
v^{m}(0)=v_{0}^{m} .
\end{array}\right.
$$

Putting $v^{m}=\sum_{j=1}^{m} \beta_{j}(t) u^{j}$ and $w=u^{i}$ for $i=1,2, \ldots, m,(20)$ is equivalent to the linear differential system respect to $t$ :

$$
\left\{\begin{array}{l}
\operatorname{Re} \frac{d \beta}{d t}=-(1-\alpha) A^{-1} B \beta-A^{-1} d \\
\beta_{i}(0)=\left(v_{0}, u^{i}\right), \quad i=1, \ldots, m
\end{array}\right.
$$

where, we have denoted

$$
A=\left(a_{i, j}\right)_{i, j=1}^{m}, \quad B=\left(b_{i, j}\right)_{i, j=1}^{m}, \quad d=\left(d_{i}\right)_{i=1}^{m}, \quad \beta=\left(\beta_{i}\right)_{i=1}^{m}
$$

with

$$
\begin{gathered}
a_{i, j}=\left(r^{1 / 2} u^{j}, r^{1 / 2} u^{i}\right), \quad b_{i, j}=\left(r^{1 / 2} u_{r}^{j}, r^{1 / 2} u_{r}^{i}\right), \\
d_{i}=\left(r^{1 / 2} \sigma_{2}^{m}, r^{1 / 2} u_{r}^{i}\right)-\left(r^{1 / 2} f_{m}, r^{1 / 2} u^{i}\right) .
\end{gathered}
$$

Notice that $A$ is symmetric and positive defined, because of

$$
\sum_{i, j=1}^{m} a_{i, j} \xi_{i} \xi_{j} \geq \sum_{i=1}^{m} \frac{1}{\lambda_{i}}\left|\xi_{i}\right|^{2} \geq \frac{1}{\lambda_{m}} \sum_{i}\left|\xi_{i}\right|^{2} \quad \forall \xi \in \mathbb{R}^{m} .
$$

Since $(1-\alpha) A^{-1} B \in \mathbb{R}^{m \times m}$ and $A^{-1} d \in C([0, T])^{m}$, there exists a unique solution $\beta \in C^{1}([0, T])^{m}$ of (21) and, therefore, a unique solution $v^{m}$ of (20) (which depends continuously on $\bar{v}^{m}, \sigma_{1}^{m}$ and $\left.\sigma_{2}^{m}\right)$. 
Then, we have rewritten (13)-(16) as the fixed point equation:

$$
\bar{v}^{m} \in C\left([0, T] ; V^{m}\right) \mapsto\left(\sigma_{1}^{m}, \sigma_{2}^{m}\right) \in C^{1}\left([0, T] ; L^{2}\right) \mapsto v^{m} \in C^{1}\left([0, T] ; V^{m}\right) .
$$

To check hypotheses of Schauder's Theorem, we have to obtain some estimates of $v^{m}$ (independent of $\bar{v}^{m}$ ). First, we are going to obtain some estimates for $\sigma_{1}^{m}, \sigma_{2}^{m}$ (independent of $\bar{v}_{r}^{m}$ and $m$ ). Computing

$$
\left((17)-\alpha \mathrm{We}^{-2}\right)\left(\sigma_{1}^{m}-\alpha \mathrm{We}^{-1}\right)+(18)\left(1-a^{2}\right) \sigma_{2}^{m}
$$

we find:

$$
\frac{d}{d t} \Psi(r, t)+2 \mathrm{We}^{-1} \Psi(r, t)=-2 \alpha \mathrm{We}^{-2}\left(\sigma_{1}^{m}-\alpha \mathrm{We}^{-1}\right) \leq \alpha^{2} \mathrm{We}^{-3}+\mathrm{We}^{-1} \Psi(r, t)
$$

where $\Psi(r, t)=\left(\sigma_{1}^{m}-\alpha \mathrm{We}^{-1}\right)^{2}+\left(1-a^{2}\right)\left(\sigma_{2}^{m}\right)^{2}$. Then,

$$
\frac{d}{d t} \Psi(r, t)+\mathrm{We}^{-1} \Psi(r, t) \leq \alpha^{2} \mathrm{We}^{-3},
$$

hence

$$
\Psi(r, t) \leq \Psi(r, 0) e^{-t / \mathrm{We}}+\alpha^{2} \mathrm{We}^{-2}\left(1-e^{-t / \mathrm{We}}\right), \quad \forall t \in[0, T], \text { a.e. } r \in(0, R) .
$$

Consequently,

$$
\left\|\sigma_{i}^{m}\right\|_{L^{\infty}\left(L^{\infty}\right)} \leq C \quad \text { for } i=1,2,
$$

where $C=C\left(\alpha, a\right.$, We, $\left.\left\|\sigma_{i, 0}\right\|_{L^{\infty}}\right)>0$, but $C$ is independent of $\bar{v}^{m}$ (and also of $m$ ).

Now, we can find estimates for $v^{m}$ (independent of $\bar{v}^{m}$ ). Indeed, taking $w=v^{m}$ in (20), one finds

$$
\frac{\operatorname{Re}}{2} \frac{d}{d t}\left\|r^{1 / 2} v^{m}\right\|^{2}+(1-\alpha)\left\|r^{1 / 2} v_{r}^{m}\right\|^{2}=-\left(r^{1 / 2} \sigma_{2}^{m}, r^{1 / 2} v_{r}\right)+\left(r^{1 / 2} f_{m}, r^{1 / 2} v\right) .
$$

This, together with (22), leads to the following estimate:

$$
\left\|r^{1 / 2} v^{m}\right\|_{L^{\infty}\left(L^{2}\right)}+\left\|r^{1 / 2} v_{r}^{m}\right\|_{L^{2}\left(L^{2}\right)} \leq C .
$$

Here $C$ depends on $\alpha$, Re, We, $\left\|\sigma_{i}^{m}\right\|_{L^{\infty} L^{\infty}},\left\|r^{1 / 2} v_{0}\right\|,\|r f\|_{L^{2}\left(V^{\prime}\right)}$, but is independent of $\bar{v}^{m}$ (and $m)$.

Since

$$
\frac{1}{\lambda_{m}} \sum_{j=1}^{m}\left|\beta_{j}(t)\right|^{2} \leq\left\|r^{1 / 2} v^{m}(t)\right\|^{2}
$$

using $(23)$ we deduce that $\left(\beta_{j}\right)$ is bounded (respect to $\bar{v}^{m}$ ) in $C([0, T])$ and, in particular,

$$
v^{m} \text { is bounded in } C\left([0, T], V^{m}\right) \text {. }
$$

Using the above estimate in (21), we have

$$
\sum_{j=1}^{m} a_{i, j} \frac{d \beta_{j}}{d t} \text { is bounded in } C([0, T]) \text {, for } 1 \leq i \leq m \text {. }
$$


Since $A$ is symmetric and defined positive (uniformly respect to $\bar{v}^{m}$ ), then $\left(\frac{d \beta_{j}}{d t}\right)$ is bounded in $C([0, T])$ for $1 \leq j \leq m$. So that

$$
\partial_{t} v^{m} \text { is bounded in } C\left([0, T], V^{m}\right) \text {. }
$$

¿From (24) and (25), we deduce that $v^{m}$ is bounded in $C^{1}\left([0, T], V^{m}\right)$.

Now, we can apply Schauder's Theorem. Indeed, there exists $C>0$ (independent of $\bar{v}^{m} \in$ $\left.C\left([0, T], V^{m}\right)\right)$ such that

$$
\left\|v^{m}\right\|_{C^{1}\left(V^{m}\right)} \leq C .
$$

If we denote $B$ (resp. $\left.B_{1}\right)$ the closed ball in $C\left([0, T], V^{m}\right)$ (resp. $\left.C^{1}\left([0, T], V^{m}\right)\right)$ with radius $C$, then the map $\bar{v}^{m} \in B \mapsto v^{m} \in B_{1}$ is well defined and continuous. Thanks to Ascoli-Arzelá Theorem, the map $\bar{v}^{m} \in B \mapsto v^{m} \in B$ is continuous and compact. Therefore, there exists a fixed point $v^{m}$, and consequently $\left(v^{m}, \sigma_{1}^{m}, \sigma_{2}^{m}\right)$ is an $m$-th approximate solution.

\section{Third step: Pass to the limit in (13)-(16) (by compactness).}

First, we are going to obtain estimates for $\left(v^{m}, \sigma_{1}^{m}, \sigma_{2}^{m}\right)$, independent of $m$. We have already obtained estimates (22) and (23) in the above step, but these estimates are not suffice to control the pass to the limit, in the nonlinear terms $v_{r}^{m} \sigma_{i}^{m}(i=1,2)$. To solve this, one possibility is to find better bounds.

We denote the $r$-derivative of an equation with the index $r$. Now, we compute

$$
\left(((14) \times r)_{r}, r^{-1}\left(r \sigma_{1}^{m}\right)_{r}\right)+\left(((15) \times r)_{r},\left(1-a^{2}\right) r^{-1}\left(r \sigma_{2}^{m}\right)_{r}\right) .
$$

On the other hand, writing (13) as

$$
\left(\operatorname{Re} \partial_{t}\left(r v^{m}\right)-(1-\alpha)\left(r v_{r}^{m}\right)_{r}, w\right)=\left\langle\left(r \sigma_{2}^{m}\right)_{r}, w\right\rangle+\left(r^{1 / 2} f_{m}, r^{1 / 2} w\right) \quad \forall w \in V^{m}
$$

we compute

$$
\left((27),-\frac{\alpha\left(1-a^{2}\right)}{\mathrm{We}} r^{-1}\left(r v_{r}^{m}\right)_{r}\right)+\left((27), \frac{\alpha\left(1-a^{2}\right)}{\mathrm{We}} \partial_{t} v^{m}\right) .
$$

Notice that $r^{-1}\left(r v_{r}^{m}\right)_{r} \in V^{m}$, thanks to the choice of the special basis. 
Adding (26) and (28), many important terms "difficult to handle" are cancelled and we obtain

$$
\left\{\begin{aligned}
\frac{1}{2} \frac{d}{d t} & \left\{\frac{1-a^{2}}{\mathrm{We}} \alpha(2-\alpha)\left\|r^{1 / 2} v_{r}^{m}\right\|^{2}+\left\|r^{-1 / 2}\left(r \sigma_{1}^{m}\right)_{r}\right\|^{2}+\left(1-a^{2}\right)\left\|r^{-1 / 2}\left(r \sigma_{2}^{m}\right)_{r}\right\|^{2}\right\} \\
& +\frac{1-a^{2}}{\mathrm{We}} \alpha(1-\alpha)\left\|r^{-1 / 2}\left(r v_{r}^{m}\right)_{r}\right\|^{2}+\frac{1-a^{2}}{\mathrm{We}} \alpha \operatorname{Re}\left\|r^{1 / 2} \partial_{t} v^{m}\right\|^{2} \\
& +\frac{1}{\mathrm{We}}\left\|r^{-1 / 2}\left(r \sigma_{1}^{m}\right)_{r}\right\|^{2}+\frac{1-a^{2}}{\mathrm{We}}\left\|r^{-1 / 2}\left(r \sigma_{2}^{m}\right)_{r}\right\|^{2} \\
& =-\frac{1-a^{2}}{\mathrm{We}} \alpha\left(r^{1 / 2} f^{m}, r^{-1 / 2}\left(r v_{r}^{m}\right)_{r}\right)+\left(1-a^{2}\right)\left(r^{1 / 2} \sigma_{2}^{m} v_{r r}^{m}, r^{-1 / 2}\left(r \sigma_{1}^{m}\right)_{r}\right) \\
& -\left(1-a^{2}\right)\left(r^{1 / 2} \sigma_{1}^{m} v_{r r}^{m}, r^{-1 / 2}\left(r \sigma_{2}^{m}\right)_{r}\right)+\frac{1-a^{2}}{\mathrm{We}} \alpha\left(r^{-1 / 2}\left(r \sigma_{2}^{m}\right)_{r}, r^{1 / 2} \partial_{t} v^{m}\right) \\
& +\frac{1-a^{2}}{\mathrm{We}} \alpha\left(r^{1 / 2} f^{m}, r^{1 / 2} \partial_{t} v^{m}\right) .
\end{aligned}\right.
$$

Using (8) and (22), the right-hand side of the above equality can be bounded by

$$
\begin{aligned}
& \frac{1-a^{2}}{2 \mathrm{We}} \alpha(1-\alpha)\left\|r^{-1 / 2}\left(r v_{r}^{m}\right)_{r}\right\|^{2}+\frac{1-a^{2}}{2 \mathrm{We}} \alpha \operatorname{Re}\left\|r^{1 / 2} \partial_{t} v^{m}\right\|^{2} \\
& +C\left(\left\|r^{-1 / 2}\left(r \sigma_{1}^{m}\right)_{r}\right\|^{2}+\left\|r^{-1 / 2}\left(r \sigma_{2}^{m}\right)_{r}\right\|^{2}+\left\|r^{1 / 2} f^{m}\right\|^{2}\right) .
\end{aligned}
$$

Thanks to Gronwall's Lemma, since $r^{1 / 2} f^{m}$ is bounded in $L^{2}\left(L^{2}\right)$ and $r^{1 / 2}\left(v_{0}^{m}\right)_{r}$ and $r^{-1 / 2}\left(r \sigma_{i, 0}\right)_{r}$ are bounded in $L^{2}$ (because $v_{0} \in V$ and $\sigma_{i, 0} \in W, i=1,2$ ), we deduce that there exists $C>0$ such that

$$
\begin{gathered}
\left\|r^{1 / 2} v_{r}^{m}\right\|_{L^{\infty}\left(L^{2}\right)} \leq C, \\
\left\|r^{-1 / 2}\left(r v_{r}^{m}\right)_{r}\right\|_{L^{2}\left(L^{2}\right)} \leq C \quad\left(\text { i.e. }\left\|v_{r}^{m}\right\|_{L^{2}(W)} \leq C\right), \\
\left\|r^{1 / 2} \partial_{t} v^{m}\right\|_{L^{2}\left(L^{2}\right)} \leq C \quad\left(\text { i.e. }\left\|\partial_{t}\left(r^{1 / 2} v^{m}\right)\right\|_{L^{2}\left(L^{2}\right)} \leq C\right)
\end{gathered}
$$

and

$$
\left.\left\|r^{-1 / 2}\left(r \sigma_{i}^{m}\right)_{r}\right\|_{L^{\infty}\left(L^{2}\right)} \leq C \quad \text { (i.e. }\left\|\sigma_{i}^{m}\right\|_{L^{\infty}(W)} \leq C\right) \quad i=1,2 .
$$

Thanks to Lemma 4, (30) and (32) imply

$$
\begin{gathered}
\left\|r^{-1 / 2} v_{r}^{m}\right\|_{L^{2}\left(L^{2}\right)} \leq C, \quad\left\|r^{-1 / 2} \sigma_{i}^{m}\right\|_{L^{\infty}\left(L^{2}\right)} \leq C, \quad i=1,2, \\
\left\|r^{1 / 2} v_{r}^{m}\right\|_{L^{2}\left(H^{1}\right)} \leq C \quad \text { and } \quad\left\|r^{1 / 2} \sigma_{i}^{m}\right\|_{L^{\infty}\left(H^{1}\right)} \leq C, \quad i=1,2,
\end{gathered}
$$

Moreover, multiplying (14) and (15) by $r^{-1 / 2}$ and taking into account (33), we obtain

$$
\left\|\partial_{t}\left(r^{-1 / 2} \sigma_{i}^{m}\right)\right\|_{L^{2}\left(L^{2}\right)} \leq C, \quad i=1,2 .
$$


Estimates (22), (23), (29) - (35) can be used to prove the existence of subsequences (again indexed with $m$ ) such that

$$
\begin{aligned}
& v^{m} \rightarrow v \quad \text { weakly in } L^{2}\left(H_{R}^{1}\right), \\
& v_{r}^{m} \rightarrow v_{r} \quad \text { weakly in } L^{2}(W), \\
& r^{1 / 2} v^{m} \rightarrow r^{1 / 2} v \quad \text { weakly } \star \text { in } L^{\infty}\left(L^{2}\right) \text { and weakly in } L^{2}\left(H_{R}^{1}\right) \text {, } \\
& r^{1 / 2} v_{r}^{m} \rightarrow r^{1 / 2} v_{r} \quad \text { weakly } \star \text { in } L^{\infty}\left(L^{2}\right) \text { and weakly in } L^{2}\left(H^{1}\right), \\
& \partial_{t}\left(r^{1 / 2} v^{m}\right) \rightarrow \partial_{t}\left(r^{1 / 2} v\right) \quad \text { weakly in } L^{2}\left(L^{2}\right), \\
& \sigma_{i}^{m} \rightarrow \sigma_{i} \quad \text { weakly } \text { in } L^{\infty}\left(L^{\infty} \cap W\right) \quad i=1,2, \\
& r^{1 / 2} \sigma_{i}^{m} \rightarrow r^{1 / 2} \sigma_{i} \quad \text { weakly } \text { in } L^{\infty}\left(H^{1}\right) \quad i=1,2, \\
& \partial_{t}\left(r^{-1 / 2} \sigma_{i}^{m}\right) \rightarrow \partial_{t}\left(r^{-1 / 2} \sigma_{i}\right) \quad \text { weakly in } L^{2}\left(L^{2}\right) \quad i=1,2 .
\end{aligned}
$$

¿From well know compactness results, in particular, one has

$$
r^{1 / 2} v^{m} \rightarrow r^{1 / 2} v \quad \text { and } \quad r^{1 / 2} \sigma_{i}^{m} \rightarrow r^{1 / 2} \sigma_{i} \quad \text { strongly in } L^{2}\left(L^{2}\right), \quad i=1,2 .
$$

The weak convergences are suffice to pass to the limit in (13) and (27), but the strong convergence is necessary to pass to the limit in $(14) \times r$ and $(15) \times r$, on account of terms $r^{1 / 2} \sigma_{i}^{m} r^{1 / 2} v_{r}^{m}$. In particular, $\left(v, \sigma_{1}, \sigma_{2}\right)$ verifies

$$
\left(\operatorname{Re} r \partial_{t} v-(1-\alpha)\left(r v_{r}\right)_{r}-\left(r \sigma_{2}\right)_{r}-r f, w\right)=0 \quad \forall w \in V .
$$

By a standard density argument, the above equality is also verified for all $w \in L^{2}$, hence one has

$$
\operatorname{Re} \partial_{t} v-(1-\alpha) \frac{1}{r}\left(r v_{r}\right)_{r}=\frac{1}{r}\left(r \sigma_{2}\right)_{r}+f \quad \text { a.e. in }(0, R) \times(0, T) .
$$

Multiplying (37) by $w \in H$, one has

$$
\partial_{t} v \in L^{2}\left(H^{\prime}\right)
$$

In particular, $v \in C\left(H^{\prime}\right)$.

On the other hand, from (32) and (35) we deduce that $\sigma_{i}^{m}$ is relativily compact in $C\left([0, T], H^{-1}\right)$ and from (23) and (31) we obtain that $r^{1 / 2} v^{m}$ is relativily compact in $C\left([0, T], H^{-1}\right)$. Then, we can pass to the limit in the initial conditions.

The Theorem 2 is proved.

\section{The proof of Theorem 1}

Let us choose sequences $\left(v_{0}^{\varepsilon}\right),\left(\sigma_{i, 0}^{\varepsilon}\right)(i=1,2)$ and $\left(f^{\varepsilon}\right)$ such that

$$
v_{0}^{\varepsilon} \in V, \quad \sigma_{i, 0}^{\varepsilon} \in L^{\infty} \cap W \quad i=1,2, \quad r^{1 / 2} f^{\varepsilon} \in L^{2}\left(L^{2}\right), \quad \forall \varepsilon>0
$$


and

$$
\begin{array}{ll}
v_{0}^{\varepsilon} \rightarrow v_{0} & \text { strongly in } H, \\
\sigma_{i, 0}^{\varepsilon} \rightarrow \sigma_{i, 0} & \text { weakly } \star \text { in } L^{\infty} \text { and a.e. in }(0, R), \\
r f^{\varepsilon} \rightarrow r f & \text { strongly in } L^{2}\left(V^{\prime}\right) .
\end{array}
$$

For each $\varepsilon>0$, we consider $\left(v^{\varepsilon}, \sigma_{1}^{\varepsilon}, \sigma_{2}^{\varepsilon}\right)$ a regular solution of (1)-(5) associated to the data $v_{0}^{\varepsilon}, \sigma_{i, 0}^{\varepsilon}$, $f^{\varepsilon}$ furnished by Theorem 2. In particular, $\left(v^{\varepsilon}, \sigma_{1}^{\varepsilon}, \sigma_{2}^{\varepsilon}\right)$ is a weak solution of (1)-(5):

$$
\begin{aligned}
& \operatorname{Re}\left(\partial_{t}\left(r^{1 / 2} v^{\varepsilon}\right), r^{1 / 2} w\right)+(1-\alpha)\left(r^{1 / 2} v_{r}^{\varepsilon}, r^{1 / 2} w_{r}\right) \\
& \quad=-\left(r^{1 / 2} \sigma_{2}^{\varepsilon}, r^{1 / 2} w_{r}\right)+\left(r f^{\varepsilon}, w\right) \quad \forall w \in V, \text { a.e. in }(0, T), \\
& \partial_{t} \sigma_{1}^{\varepsilon}+\mathrm{We}^{-1} \sigma_{1}^{\varepsilon}=\left(1-a^{2}\right) \sigma_{2}^{\varepsilon} v_{r}^{\varepsilon} \quad \text { a.e. in }(0, R) \times(0, T), \\
& \partial_{t} \sigma_{2}^{\varepsilon}+\mathrm{We}^{-1} \sigma_{2}^{\varepsilon}=\alpha \mathrm{We}^{-1} v_{r}^{\varepsilon}-\sigma_{1}^{\varepsilon} v_{r}^{\varepsilon} \quad \text { a.e. in }(0, R) \times(0, T), \\
& \left.\left(r^{1 / 2} v^{\varepsilon}\right)\right|_{t=0}=r^{1 / 2} v_{0}^{\varepsilon},\left.\quad\left(r^{1 / 2} \sigma_{i}^{\varepsilon}\right)\right|_{t=0}=r^{1 / 2} \sigma_{i, 0}^{\varepsilon} \quad i=1,2 .
\end{aligned}
$$

Proceeding as in section 4, we can prove the estimates (22) and (23) (respect to $\varepsilon$ ), that is

$$
\left\|\sigma_{i}^{\varepsilon}\right\|_{L^{\infty}\left(L^{\infty}\right)} \leq C, \quad\left\|r^{1 / 2} v^{\varepsilon}\right\|_{L^{\infty}\left(L^{2}\right)} \leq C, \quad\left\|r^{1 / 2} v_{r}^{\varepsilon}\right\|_{L^{2}\left(L^{2}\right)} \leq C .
$$

Considering these estimates in (40), (41), one has

$$
\left\|\partial_{t}\left(r^{1 / 2} \sigma_{i}^{\varepsilon}\right)\right\|_{L^{2}\left(L^{2}\right)} \leq C, \quad i=1,2 .
$$

In addition, the estimate

$$
\left\|\partial_{t}\left(r^{1 / 2} v^{\varepsilon}\right)\right\|_{L^{2}\left(H_{R}^{-1}\right)} \leq C
$$

can be proved, using the following

Lemma 6 If $w \in H_{R}^{1}$ then $r^{-1 / 2} w \in V$ and $\left\|r^{-1 / 2} w\right\|_{V} \leq C\|w\|_{H_{R}^{1}}$.

Proof:

By definition of $V, r^{-1 / 2} w \in V$ if only if $r^{1 / 2}\left(r^{-1 / 2} w\right)_{r}=w_{r}-\frac{1}{2} r^{-1} w \in L^{2}$. Since $w_{r} \in L^{2}$, it suffices to prove that $r^{-1} w \in L^{2}$ and $\left\|r^{-1} w\right\| \leq C\left\|w_{r}\right\|$. Indeed, we can write

$$
\left(r^{-1} w\right)^{2}(r)=-2 \int_{r}^{R} s^{-1} w(s)\left(s^{-1} w(s)\right)_{s} d s .
$$

Integrating in $r \in(0, R)$ and using Fubini's Theorem, we obtain

$$
\left\|r^{-1} w\right\|^{2}=-2 \int_{0}^{R} w(r)\left(r^{-1} w(r)\right)_{r} d r=2\left\|r^{-1} w\right\|^{2}-2 \int_{0}^{R} r^{-1} w(r) w_{r}(r) d r .
$$

Therefore,

$$
\left\|r^{-1} w\right\|^{2}=2 \int_{0}^{R} r^{-1} w(r) w_{r}(r) d r \leq 2\left\|r^{-1} w\right\| \cdot\left\|w_{r}\right\|,
$$


and $\left\|r^{-1} w\right\| \leq 2\left\|w_{r}\right\|$ holds.

Thanks to the estimates (43)-(45), we can pass to the limit in (39) and in the linear terms of (40)-(41). Moreover, the initial conditions are verified. However, the weak convergences are not enough to pass to the limit in the nonlinear terms $\sigma_{i}^{\varepsilon} v_{r}^{\varepsilon}$ of (40)-(41). To do it, we will prove that $\left(r^{1 / 2} v^{\varepsilon}, r^{1 / 2} \sigma_{1}^{\varepsilon}, r^{1 / 2} \sigma_{2}^{\varepsilon}\right)$ is a Cauchy sequence in an addecuate norm.

We consider the difference between $(39)_{\varepsilon}-(41)_{\varepsilon}$ and $(39)_{\eta}-(41)_{\eta}$. Taking $w=\frac{\alpha}{\mathrm{We}}\left(v^{\varepsilon}-v^{\eta}\right)$ as test function in $(39)_{\varepsilon}-(39)_{\eta}$, computing the scalar products in $L^{2}$ of $\left((40)_{\varepsilon}-(40)_{\eta}\right) \times r$ and $\left((41)_{\varepsilon}-(41)_{\eta}\right) \times r$ respectively by $\frac{1}{1-a^{2}} r^{1 / 2}\left(\sigma_{1}^{\varepsilon}-\sigma_{1}^{\eta}\right)$ and $r^{1 / 2}\left(\sigma_{2}^{\varepsilon}-\sigma_{2}^{\eta}\right)$, and adding all the terms, we obtain

$$
\left\{\begin{aligned}
\frac{1}{2} \frac{d}{d t} & \left\{\frac{\alpha \operatorname{Re}}{\mathrm{We}}\left\|r^{1 / 2}\left(v^{\varepsilon}-v^{\eta}\right)\right\|^{2}+\frac{1}{1-a^{2}}\left\|r^{1 / 2}\left(\sigma_{1}^{\varepsilon}-\sigma_{1}^{\eta}\right)\right\|^{2}+\left\|r^{1 / 2}\left(\sigma_{2}^{\varepsilon}-\sigma_{2}^{\eta}\right)\right\|^{2}\right\} \\
& +\frac{\alpha(1-\alpha)}{\mathrm{We}}\left\|r^{1 / 2}\left(v_{r}^{\varepsilon}-v_{r}^{\eta}\right)\right\|^{2}+\frac{1}{\mathrm{We}}\left(\frac{1}{1-a^{2}}\left\|r^{1 / 2}\left(\sigma_{1}^{\varepsilon}-\sigma_{1}^{\eta}\right)\right\|^{2}+\left\|r^{1 / 2}\left(\sigma_{2}^{\varepsilon}-\sigma_{2}^{\eta}\right)\right\|^{2}\right) \\
& =\frac{\alpha}{\mathrm{We}}\left(r^{1 / 2}\left(\sigma_{2}^{\varepsilon}-\sigma_{2}^{\eta}\right), r^{1 / 2}\left(v_{r}^{\varepsilon}-v_{r}^{\eta}\right)\right)+\frac{\alpha}{\mathrm{We}}\left(r\left(f^{\varepsilon}-f^{\eta}\right),\left(v^{\varepsilon}-v^{\eta}\right)\right) \\
& +\left(\sigma_{2}^{\eta} r^{1 / 2}\left(v_{r}^{\varepsilon}-v_{r}^{\eta}\right), r^{1 / 2}\left(\sigma_{1}^{\varepsilon}-\sigma_{1}^{\eta}\right)\right)+\frac{\alpha}{\mathrm{We}}\left(r^{1 / 2}\left(v_{r}^{\varepsilon}-v_{r}^{\eta}\right), r^{1 / 2}\left(\sigma_{1}^{\varepsilon}-\sigma_{1}^{\eta}\right)\right) \\
& \left.-\left(\sigma_{1}^{\eta} r^{1 / 2}\left(v_{r}^{\varepsilon}-v_{r}^{\eta}\right)\right), r^{1 / 2}\left(\sigma_{2}^{\varepsilon}-\sigma_{2}^{\eta}\right)\right) .
\end{aligned}\right.
$$

Here, we have added and subtracted $\sigma_{2}^{\eta} v_{r}^{\varepsilon}$ in $(40)_{\varepsilon}-(40)_{\eta}$ and $\sigma_{1}^{\eta} v_{r}^{\varepsilon}$ in $(41)_{\varepsilon}-(41)_{\eta}$ respectively. It must be emphasized that, in the previous manipulations, those terms "difficult to handle", i.e. the terms associated to

$$
\left(1-a^{2}\right)\left(\sigma_{2}^{\varepsilon}-\sigma_{2}^{\eta}\right) v_{r}^{\varepsilon} \quad \text { and } \quad-\left(\sigma_{1}^{\varepsilon}-\sigma_{1}^{\eta}\right) v_{r}^{\varepsilon}
$$

have disappeared. The right-hand side of (46) is bounded by

$$
\begin{aligned}
& C\left(\left\|r^{1 / 2}\left(v_{r}^{\varepsilon}-v_{r}^{\eta}\right)\right\| \cdot\left\|r^{1 / 2}\left(\sigma_{1}^{\varepsilon}-\sigma_{1}^{\eta}\right)\right\|+\left\|r^{1 / 2}\left(v_{r}^{\varepsilon}-v_{r}^{\eta}\right)\right\| \cdot\left\|r^{1 / 2}\left(\sigma_{2}^{\varepsilon}-\sigma_{2}^{\eta}\right)\right\|\right. \\
& \left.\quad+\left\|r\left(f^{\varepsilon}-f^{\eta}\right)\right\|_{V^{\prime}} \cdot\left\|\left(v^{\varepsilon}-v^{\eta}\right)\right\|_{V}\right) .
\end{aligned}
$$

Then, we arrive at

$$
\left\{\begin{aligned}
\frac{d}{d t}\{ & \left.\frac{\alpha \operatorname{Re}}{\mathrm{We}}\left\|r^{1 / 2}\left(v^{\varepsilon}-v^{\eta}\right)\right\|^{2}+\frac{1}{1-a^{2}}\left\|r^{1 / 2}\left(\sigma_{1}^{\varepsilon}-\sigma_{1}^{\eta}\right)\right\|^{2}+\left\|r^{1 / 2}\left(\sigma_{2}^{\varepsilon}-\sigma_{2}^{\eta}\right)\right\|^{2}\right\} \\
& +\frac{\alpha(1-\alpha)}{\mathrm{We}}\left\|r^{1 / 2}\left(v_{r}^{\varepsilon}-v_{r}^{\eta}\right)\right\|^{2} \leq C_{1}\left\|r\left(f^{\varepsilon}-f^{\eta}\right)\right\|_{V^{\prime}}^{2} \\
& +C_{2}\left[\frac{\alpha \operatorname{Re}}{\mathrm{We}}\left\|r^{1 / 2}\left(v^{\varepsilon}-v^{\eta}\right)\right\|^{2}+\frac{1}{1-a^{2}}\left\|r^{1 / 2}\left(\sigma_{1}^{\varepsilon}-\sigma_{1}^{\eta}\right)\right\|^{2}+\left\|r^{1 / 2}\left(\sigma_{2}^{\varepsilon}-\sigma_{2}^{\eta}\right)\right\|^{2}\right]
\end{aligned}\right.
$$


¿From Gronwall's Lemma, we obtain

$$
\begin{aligned}
& \frac{\alpha \operatorname{Re}}{\mathrm{We}}\left\|r^{1 / 2}\left(v^{\varepsilon}-v^{\eta}\right)\right\|_{L^{\infty}\left(L^{2}\right)}^{2}+\frac{1}{1-a^{2}}\left\|r^{1 / 2}\left(\sigma_{1}^{\varepsilon}-\sigma_{1}^{\eta}\right)\right\|_{L^{\infty}\left(L^{2}\right)}^{2}+\left\|r\left(\sigma_{2}^{\varepsilon}-\sigma_{2}^{\eta}\right)\right\|_{L^{\infty}\left(L^{2}\right)}^{2} \\
& \quad \leq \frac{\alpha \operatorname{Re}}{\mathrm{We}}\left\|r^{1 / 2}\left(v_{0}^{\varepsilon}-v_{0}^{\eta}\right)\right\|_{L^{2}}^{2}+\frac{1}{1-a^{2}}\left\|r^{1 / 2}\left(\sigma_{1,0}^{\varepsilon}-\sigma_{1,0}^{\eta}\right)\right\|_{L^{2}}^{2}+\left\|r^{1 / 2}\left(\sigma_{2,0}^{\varepsilon}-\sigma_{2,0}^{\eta}\right)\right\|_{L^{2}}^{2} \\
& \quad+C\left\|r\left(f^{\varepsilon}-f^{\eta}\right)\right\|_{L^{2}\left(V^{\prime}\right)}^{2} .
\end{aligned}
$$

Therefore, thanks to (38), $\left\{r^{1 / 2} v^{\varepsilon}\right\}$ and $\left\{r^{1 / 2} \sigma_{i}^{\varepsilon}\right\}(i=1,2)$ are Cauchy sequences in $L^{\infty}\left(L^{2}\right)$. Coming back to (47), we also obtain that $\left\{r^{1 / 2} v_{r}^{\varepsilon}\right\}$ is a Cauchy sequence in $L^{2}\left(L^{2}\right)$.

Notice that, in particular, the above argument implies uniqueness of the weak solution.

The strong convergences of $r^{1 / 2} \sigma_{i}^{\varepsilon}$ in $L^{\infty}\left(L^{2}\right)$ or $r^{1 / 2} v_{r}^{\varepsilon}$ in $L^{2}\left(L^{2}\right)$, are enough to take limits in $(40) \times r^{1 / 2}$ and $(41) \times r^{1 / 2}$. Finally, from estimates (43)-(45), one has that the sequence $\left\{r^{1 / 2} \sigma_{i}^{\varepsilon}\right\}$ is relatively compact in $C\left([0, T], H^{-1}\right)$ and $\left\{r^{1 / 2} v^{\varepsilon}\right\}$ in $C\left([0, T], H_{R}^{-1}\right)$, hence the initial conditions holds.

Remark: The Cauchy argument used in the above proof, can not be made directly with a Galerking approximation. From this fact, we have had to prove the regularity result (Theorem 2) before that Theorem 1.

Remark: When $a=1$ or $a=-1$, results like of this paper can be proved more easily. Now, $\sigma_{2}$ is uncoupled respect to $\left(v, \sigma_{1}\right)$, which is the solution of a linear system. The principal disavantage is that now one can not prove the $L^{\infty}$ estimates for $\sigma_{1}$. In this new system, the energy estimates says us that

$$
\begin{aligned}
& r^{1 / 2} \sigma_{1}^{m} \text { is bounded in } L^{\infty}\left(L^{2}\right), \\
& r^{1 / 2} v^{m} \text { is bounded in } L^{\infty}\left(L^{2}\right), \\
& r^{1 / 2} v_{r}^{m} \text { is bounded in } L^{2}\left(L^{2}\right) .
\end{aligned}
$$

Therefore a standard compactness argument leads to the existence (and uniqueness) of the weak solution.

Acknowledgment: The authors are indebted to E. Fernández-Cara (University of Sevilla), for several fruitful discussions and specially for their introduction in this subject.

\section{References}

[1] G. Astarita, G.Marruci - Principles of Non-Newtonian Fluid Mechanics, McGraw Hill, New York, 1974. 
[2] D. Guedes de Figueiredo - Positive solutions of Semilinear elliptic problems, Lectures Notes in Mathematics, Vol. 957, p. 34-88, 1982.

[3] E. Fernández-Cara, F. Guillen, R. R. Ortega - Some theoretical results concerning Non-Newtonian fluids of the Oldroyd kind, Ann. Scuola Norm. Sup. Pisa Cl. Sci. (4), Vol. XXVI, p. 1-29, 1998.

[4] C. Guillopé, J.C. SAUt - Existence results for the flow of viscoelastic fluids with a differential constitutive law, Nonlinear Anal. Vol. 15, No.9, p. 849-869, 1990.

[5] C. Guillopé, J.C. SAUT - Global existence and one-dimensional nonlinear stability of shearing motions of viscoelastic fluids of Oldroyd type, Math. Mod. Numer. Anal. Vol. 24, No.3, p. 369-401, 1990.

[6] J.G. OldRoyd - On the formulation of rheological equations of state, Proc. Roy. Soc. London Ser. A 200, p. 523-541, 1950.

[7] R.R. Ortega - Thesis, Univerity of Seville (Spain), 1995.

[8] M. Renardy, W.J. Hrusa, J.A. Nohel - Mathematical problems in viscoelasticity, Longman, London, 1987. 